\title{
184. Plastische Deckung problematischer Weichteildefekte
}

\author{
L. Zwank und P. Eckert \\ Kliniken der Stadt Saarbrücken „Winterberg“, Akademisches Krankenhaus, Chirurgische Klinik, \\ Theodor-Heuß-Straße 120, D-6600 Saarbrücken 6
}

\section{Closure of Problematic Soft-Tissue and Skin Defects by Plastic Surgery}

Summary. The advent of microvascular free flaps has provided us with the possibility of healing defects that could not be closed by other previous procedures. In correspondence with depth and localization of the defect, we transplant temporal flaps, as well as latissimus dorsi, deltopectoral, inguinal, dorsum of the foot, and pulpal flaps. With examples from our clinic, we show the use of the above-mentioned procedure in cases for which other techniques have been unsuccessful. Only one microvascular transplant out of 30 did not heal because we placed it on a case of florid osteomyelitis.

Key words: Microvascular free flaps - Soft tissue defect - Latissimus dorsi flap - Osteomyelitis.

Zusammenfassung. Durch mikrovasculär gestielte Hautweichteillappen können Gewebsdefekte ausgeheilt werden, bei denen zahlreiche andere vorausgegangene Deckungsversuche mißlangen. Je nach Tiefe und Sitz des zu verschließenden Defektes transplantieren wir Temporalislappen, Latissimus-dorsi-Lappen, deltopectorale Lappen, Leistenlapen, Fußrückenlappen und Pulpalappen. Es werden mehrere klinische Beispiele gezeigt, bei welchen herkömmliche Deckungsversuche versagten und die einzelnen obengenannten Lappen zur Anwendung kamen. Von 30 frei transplantierten mikrovasculär gestielten Hautweichteillappen heilte einer nicht ein. Er wurde über einer floriden Osteomyelitis transplantiert.

Schlüsselwörter: Freie mikrovasculäre Lappen - Weichteildefekte - Latissimus-dorsi-Lappen - Osteomyelitis.

\section{Die Kombination verschiedener Techniken zur Deckung großflächiger Defekte im Thorax-, Hals- und Axillarbereich}

\author{
F. Dinkelaker und E. Vaubel
}

Abteilung für Unfall- und Wiederherstellungschirurgie, Klinikum Steglitz der FU, Hindenburgdamm 30, D-1000 Berlin 45

\section{The Combination of Different Methods to Cover Large Defects of the Chest Wall, Axilla, and Throat}

Summary. Covering large defects of the chest wall, axilla, and throat is still a problem in reconstructive surgery. Etiologically, most of our patients have radiological ulcers of different origin. Each of the different methods of coverage is limited by its own operative technique; therefore, in some rare cases, only a combination of different methods is successful. Using some of our patients as examples, the combination of local rotation flaps, rotation of the opposite breast, and transposition of greater omentum and myocutaneous island flaps with the latissimus dorsi and rectus abdominis muscles is demonstrated.

Key words: Omentum majus - M. rectus abdominis - M. latissimus dorsi - Myocutaneous island flaps.

Zusammenfassung. Die Deckung großfächiger Defekte im Thorax-, Hals- und Axillarbereich ist auch heute noch ein Problem. Aetiologisch handelt es sich meist um Patienten mit Strahlenulcera verschiedenster Genese. Die einzelnen Methoden der Deckung haben jeweils operative Beschränkungen, so da $B$ in manchen seltenen Fällen nur die Kombination verschiedener Techniken erfolgreich ist. Anhand von Fallbeispielen wird die Kombination von lokalen Rotationslappen, von der Rotation der gesunden Brust über den Defekt, von der Omentum-Transposition und von myocutanen Insellappen mit dem M. latissimus dorsi und M. rectus abdominis im einzelnen dargestellt.

Schliisselwörter: Omentum majus - M. rectus abdominis - M. latissimus dorsi - Myocutanlappen. 